\title{
Involvement of the rat prefrontal cortex in cognitive functions: A central role for the prelimbic area
}

\author{
SYLVIE GRANON \\ Institut Pasteur, Paris, France \\ and \\ BRUNO POUCET \\ CRNC, CNRS, Marseille, France
}

\begin{abstract}
In this brief review, we address the cognitive functions of a subregion of the rat frontal cortex, the prelimbic cortex. Growing evidence suggests that the prelimbic cortex is involved in working memory, defined as the temporary storage of information required for its internal manipulation. However, several factors appear to modulate the extent to which prelimbic damage impairs performance in delayed tasks. These factors, which contribute to the overall difficulty of the task, are related to the attentional requirement of the task and to the response selection mechanisms that underlie correct performance. Impairments induced by prelimbic cortical damage are increased when the task requires the rat to consistently focus its attention on the detection of external events and when the learning rule countradicts either spontaneously used or previously learned strategies. This overall pattern of deficit suggests that the prelimbic cortex is not a pure working memory system. Rather, it subtends a wide range of processes that are required for solving difficult problems. Together with anatomical evidence, the existence of functional similarities between the prelimbic cortex of the rat and the dorsolateral prefrontal cortex of primates suggests some homology between these regions across species. Therefore, the rat prelimbic cortex appears to provide a valuable model system for studying the precursors of higher level cognitive processes in nonhuman and human primates.
\end{abstract}

While the role of the cortex in learning and memory has long been acknowledged in the rat (Lashley, 1929), it is only relatively recently that a regional analysis of its functions has been undertaken. This is especially true for the prefrontal cortex probably because its involvement in a specific learning process is hard to determine. One reason for this difficulty is that the frontal cortex is not a homogeneous area. Therefore, its damage induces mixed patterns of impaired and spared abilities depending on the exact locus of the lesion, often making difficult the interpretation of results drawn from different studies. ${ }^{1}$

In the present review, we focus on a subregion of the rat medial prefrontal cortex, the prelimbic cortex (PL), an area currently thought to be homologous to the dorsolateral prefrontal cortex of human and nonhuman primates. In the primate literature, the dorsolateral prefrontal cortex has recently received much attention with regard to its possible role in a category of high-level cognitive processes, usually referred to as executive functions. Such functions

Support for this work was provided by the Pasteur Institute and the French National Center for Scientific Research. We thank two anonymous referees for their helpful comments on an earlier version of this paper. Correspondence should be addressed to $S$. Granon, Département de Virologie Moléculaire, Institut Pasteur, 25 rue du Dr. Roux, 75075 Paris, France (e-mail: granon@pasteur.fr). are hypothesized to underlie human problem solving and refer to complex cognitive processes required to perform flexible and voluntary goal-directed behaviors based on stored information in accordance with the context (Stuss $\&$ Benson, 1983). Executive functions rely on a set of distinctive processes, such as working memory, attentional capacities, and response-selection mechanisms, all of which are necessary for successful problem solving.

Early research of the medial prefrontal cortex in rodents, which includes the prelimbic area, is consistent with at least some function in working memory (see Kolb, 1990, for review). It is not clear, however, whether its other functional properties can be described along the lines suggested by the theory of executive functions developed in the primate literature. Our purpose in this article is to attempt to bridge the rat and primate literature with regard to the putative cognitive functions of the PL. To do so, we briefly discuss evidence that human executive functions involve the dorsolateral prefrontal cortex (DLPFC). We then describe recent experimental data obtained in the rat so as to examine whether the rat PL is involved in working memory. We further ask whether, within working memory, the prelimbic function is best characterized by its involvement in a certain type of processing (e.g., maintaining information across a delay or manipulating such information to select a response) or by its involvement in 
processing certain types of information (e.g., spatial vs. nonspatial information). Lastly, we will briefly describe the results of a few experiments that have addressed the involvement of the prelimbic cortex in attentional functions.

\section{PREFRONTAL CORTEX AND EXECUTIVE FUNCTIONS IN HUMANS AND NONHUMAN PRIMATES}

As stated above, executive functions support flexible goal-directed behaviors based on stored information. Initially, relevant information must be selected from the environment through some multimodal sensorial filter (i.e., attentional mechanisms). To be internally manipulated, information then needs to be temporally organized and stored in a temporary memory buffer (i.e., working memory). Finally, appropriate decisions regarding the use of this information in this particular context must be made (i.e., response-selection function). Each of these processes seems to depend on the DLPFC, as shown by studies of patients with prefrontal lesions and by recent imaging studies as well as by behavioral and electrophysiological data from primates (Fuster, 1997; GoldmanRakic, 1990; Grafman, 1994; Miller, 1999).

Although it has long been thought that working memory processes within the frontal cortex are organized according to the nature of information being processed (e.g., with spatial information processed within the DLPFC; Goldman-Rakic, 1987), this view has recently been challenged. Specifically, recent functional brain imaging studies have suggested that the subdivision of the human prefrontal cortex in dorsal and ventral regions may be reconsidered according to the type of processing achieved in each region (D'Esposito, Aguirre, Zarahn, Ballard, Shin, $\&$ Lease, 1998; Owen et al., 1999). In other words, the function of the different prefrontal areas may depend on the type of processing performed on the information more than on the type of information (spatial vs. nonspatial information). For example, D'Esposito and collaborators (1998) showed that both spatial and nonspatial working memory tasks may result in prefrontal activation of the same regional area when tested in tasks that tap the same type of processing. In contrast, they reported that the ventral areas of the human prefrontal cortex are selectively activated when the subject only has to maintain information in working memory, whereas the dorsal areas are selectively recruited when the subject has to manipulate information in working memory in order to achieve the task.

Other studies have considered the role of the prefrontal cortex in attentional mechanisms. Attention participates in cognitive functions by preparing, facilitating, and modulating perception and action. Attention is not a unitary process since it covers a wide variety of functions (Ojemann, Buckner, Corbetta, \& Raichle, 1997), and the heterogeneity and complexity of the prefrontal cortex can provide the basis for processes under attentional control (Stuss, Shallice, Alexander, \& Picton, 1995). Evidence for a role of the prefrontal cortex in attentional function is primarily suggested by the observation that this area is the major cortical target of the main neurotransmitter ascending systems involved in arousal and attentional processes (Robbins, 1997). Furthermore, lesions of the DLPFC affect attentional abilities. Imaging studies show increased activity of this region in tasks that involve sustained or selective attention (Corbetta, Miezin, Dobmeyer, Shulman, \& Petersen, 1991; Posner \& Dehaene, 1994). Current theories view the DLPFC as a part of an attentional network, which would also involve the parietal and cingulate cortices. However, understanding the specific role of the prefrontal cortex in attentional functions is far from being an achieved goal (see Ojemann et al., 1997, for review). In particular, it is not known whether the same subregions of the prefrontal cortex are implicated in different attentional functions and, thus, in different cognitive processes.

In spite of the difficulties encountered in trying to disentangle the involvement of the DLPFC in working memory and attention in humans, recent work in monkeys provides the first physiological evidence that attentional and working memory processes are intimately related and that prefrontal cortex neurons can provide support for this relationship (Rainer, Asaad, \& Miller, 1998). Indeed, these authors reported that the same prefrontal neurons can be shown to increase their firing both during a selective attention task and during a working memory delay. It is therefore possible that the DLPFC is part of a neuronal network that is concurrently involved in these two aspects of executive functions.

\section{SPECIFICITY OF THE RAT PL IN COGNITIVE FUNCTIONS}

Although this paper focuses on functional data, we first provide some elements of neuroanatomy about the rat prefrontal cortex in general and about the prelimbic area in particular. This brief overview will point out that while some data are consistent with the existence of a homology between the primate DLPFC and the rat prelimbic area, other data rather suggest a homology between the primate DLPFC and other subareas of the rat prefrontal cortex (e.g., the anterior cingulate).

The prefrontal areas are defined by their thalamocortical, subcortical, and cortico-cortical projections, as well as by their cytoarchitecture. Consistent with the existence of an anatomical homology between the rat and the primate prefrontal cortex is the fact that the overall arrangement of the thalamocortical connections in the rat (especially with the mediodorsal nucleus, MD) parallels the arrangement observed in the primates (Kolb, 1990). On the basis of these connections, the rat prefrontal cortex has been shown to consist of two separate areas, the medial and the lateral regions. The medial prefrontal cortex (mPFC) consists of at least three different subareas, the anterior cingulate, the prelimbic area, and the infralimbic regions, respectively $(\mathrm{Cg} 1, \mathrm{Cg} 3$, and $\mathrm{IL}$ 
in Zilles's, 1985, terminology). Some anatomical studies also include the medial precentral area $(\mathrm{PrCm})$ as a part of the mPFC (Condé, Audinat, Maire-Lepoivre, \& Crépel, 1990; Preuss, 1995). These areas differ in both their pattern of connectivity and cytoarchitecture (Groenewegen, Berendse, Wolters, \& Lohman, 1990; Krettek \& Price, 1977).

In rats, the PL receives massive projections from the mediodorsal thalamic nuclei of the thalamus and afferent inputs from sensory and motor structures as well as limbic structures such as the amygdala (Krettek \& Price, 1977). Also, a direct connection with the hippocampus has been described (Jay, Glowinski, \& Thierry, 1989). In return, the PL projects to autonomic centers, the striatum, pallidum, nucleus accumbens, mediodorsal nucleus of the thalamus, and the amygdala (Sesack, Deutsch, Roth, \& Bunney, 1989). Although these anatomical studies of the mPFC suggest some homology between the rat PL and the DLPFC of primates, a number of details differ between the two species. For example, while the primate DLPFC receives inputs from the parvocellular component of the mediodorsal nucleus of the thalamus (lateral part of the MD), it is the medial segment of the MD that projects to the rat prelimbic area (Fuster, 1997; Uylings \& Van Eden, 1990). In humans, no direct connection with the hippocampus has been described. However, in monkeys, the area around the sulcus principalis has been reported to receive afferents from and send efferents to the hippocampus (Fuster, 1997). These differences, together with other considerations about connectivity, led some authors to suggest that similarities between the rat prelimbic area and the primate DLPFC do not seem to exist (Condé, Maire-Lepoivre, Audinat, \& Crépel, 1995). For Preuss (1995), the granular frontal cortex, consisting of areas 46,9 , and 8 , would be unique to primates. A further idea is that the rat anterior cingulate cortex may be the prefrontal subarea most homologous to the primate DLPFC (Uylings \& Van Eden, 1990).

Therefore, the existence of a strict anatomical homology between the rat and the primate prefrontal subareas is problematic. It is probably at the functional level that resemblances can be worked out. Because earlier lesion studies considered the rat $\mathrm{mPFC}$ as a whole, the function of its subregions was not addressed. Consistent with gross anatomical considerations, these early behavioral experiments suggested that the rat $\mathrm{mPFC}$ is involved in working memory (see Kolb, 1984, for review). In fact, it is only recently that research has focused on the role of the rat medial prefrontal subareas. In the remainder of this paper, we focus on the prelimbic area, a mPFC subarea that has received the most attention. Indeed, only a few recent studies have started to analyze the specific role of the anterior cingulate area.

Two earlier studies (Johnston, Hart, \& Howell, 1974; Larsen \& Divac, 1978) first suggested that the rat mPFC is functionally heterogeneous. In both studies, the authors used rat equivalents of typical "prefrontal tasks" used in primates (i.e., tasks requiring working memory). Larsen and Divac (1978) showed that only "pregenual lesions"that is, lesions of the structure anterior to the genu of the corpus callosum - disrupt delayed response performance in well-trained rats. The lesion involved the PL and a small anterior part of the anterior cingulate $\mathrm{Cgl}$.

Johnston et al. (1974) showed that bilateral lesions of the medial part of the frontal cortex, but not those of its lateral parts, produce severe impairments in a go/no-go task, suggesting an altered capacity of the rat to organize temporal events in a coherent manner. Such deficits can be seen as resulting from a general working memory impairment since such memory requires both maintaining information during a brief delay and temporally organizing events within this memory buffer (Pribram, Ahumada, Hartog, \& Ross, 1964). They closely resemble those described in humans (Milner, 1971) and nonhuman primates (Fuster, 1997) following dorsolateral frontal lesions. These two studies thus unambiguously show the functional heterogeneity of the rat frontal cortex. Moreover, they suggest some level of homology between the rat MPFC and the primate's DLPFC.

Recent literature fully supports the idea that the prefrontal cortex subregions differentially contribute to $\operatorname{cog}$ nitive functions (Bussey, Muir, Everitt, \& Robbins, 1997; Ragozzino, Adams, \& Kesner, 1998; Ragozzino, Wilcox, Raso, \& Kesner, 1999; Seamans, Floresco, \& Phillips, 1995 ). While damage to the rat mPFC produces working memory impairments in T-maze alternation (Thomas \& Brito, 1980), spatial delayed matching to sample in a modified version of the water maze task (Kolb, Buhrmann, McDonald, \& Sutherland, 1994), and delayed matching to position (Dunnett, 1990), lesions restricted to the PL are sufficient to produce similar behavioral effects (Brito \& Brito, 1990; Granon \& Poucet, 1995; Granon, Vidal, Thinus-Blanc, Changeux, \& Poucet, 1994; Li \& Shao, 1998; Tzschenk \& Schmidt, 1998). In contrast, lesions that spare the PL produce much less dramatic impairments (Fritts, Asbury, Horton, \& Isaac, 1998) or no impairment at all in a spatial delayed alternation task (Sánchez-Santed, de Bruin, Heinsbroek, \& Verwer, 1997). Thus, most of the behavioral effects of large mPFC lesions in working memory can be reproduced by lesions restricted to the prelimbic area of the prefrontal cortex (Brito, Thomas, \& Davis, 1982). To summarize, in contrast to neuroanatomy, functional evidence indicates a specific involvement of the rat prelimbic area in some cognitive processes that are usually associated with the primate DLPFC function.

\section{PL AND WORKING MEMORY}

There are currently two conflicting views of working memory. The first view states that working memory is merely a temporary store and thus that any lesioninduced impairment should be delay dependent. This is because increasing the delay alters the capacity of the temporary store to hold information over time. Another view, however, considers that working memory operates 
in conditional learning tasks whenever correct responses are contingent on previous events (Moscovitch \& Winocur, 1995). Seen this way, working memory is more readily defined as "working-with-memory" rather than as a simple temporary memory store. As a consequence, this conception insists on the integration of stored information and response selection mechanisms, implying that such processes should not be affected by time. According to this view, working-with-memory deficits should not be delay dependent. With regard to this point, current evidence is controversial (see discussion in Joel, Tarrasch, Feldon, \& Weiner, 1997). Prefrontal damage has been reported to produce both delay-dependent (e.g., Brito \& Brito, 1990; Delatour \& Gisquet-Verrier, 1999) and delay-independent (e.g., Delatour \& Gisquet-Verrier, 1996) memory deficits. Even though the issue of whether or not working memory implies delay-dependent effects is outside the scope of the present paper, we briefly discuss here possible explanations of such contradictory evidence since they might reveal an interesting facet of the PL in cognitive processing.

\section{Delayed Tests}

In the go/no-go task, Delatour and Gisquet-Verrier (1996) reported no detrimental effects of increasing the delay in rats with PL lesions. In this study, rats with PL lesions were initially impaired during acquisition (see also Johnston et al,, 1974, which used rats with larger mPFC lesions), but eventually recovered with extensive training. No deficit was observed when the delay was introduced after rats had recovered in the zero-delay condition. Thus, the performance of lesioned rats in go/no-go tasks does not seem to be affected by the delay. Similar lack of effect is found with lesions of the more dorsomedial part of the MPFC (Ac and PrCm, Winocur, 1991). These results suggest that the PL, as well as the more dorsal part of the mPFC, may not be always required for maintaining information in memory across long delays.

Quite the contrary, PL damage has been consistently shown to produce delay-dependent effects in spatial delayed alternation tasks (Brito \& Brito, 1990; de Brabander, de Bruin, \& Van Eden, 1991; Delatour \& GisquetVerrier, 1996; Van Haaren, de Bruin, Heinsbroek, \& Van de Poll, 1985). In this task, rats have to alternate between the two arms of a Y-maze or T-maze to find the reward. Contrary to the go/no-go task, this task emphasizes the need for spatial response selection processes, since the rat must (1) know the spatial rule to apply (i.e., to alternate), (2) store the last visited spatial location in its working memory, and (3) choose between two arms distinguishable only by their spatial characteristics. It might thus be argued that the delay-dependent prefrontal deficit observed in some situations results from the spatial nature of the response to be made since the traditional go/no-go task does not require a spatial response.

This hypothesis, however, meets several difficulties. First, it is not consistent with the results of Ragozzino et al. (1998), who tested the effect of prelimbic/infralimbic lesions on spatial working memory in a radial maze. In this study, rats were exposed twice to the same arm of the radial maze, but only the first visit to that arm was reinforced. Visits of other arms could be inserted between visits to the "target" arm so that a lag of zero to six arms was possible between the two presentations of the same arm. Performance was measured by the adaptation of rats to run slowly on the second visit relative to the first. Results showed that rats with prelimbic lesions were impaired even though the lag between the first (reinforced) and second (not reinforced) exposure to the same arm was low. This deficit increased similarly in control and lesioned animals when the time or the number of arms visited between the first and the second exposure was increased. This result is not consistent with the observation of delay-dependent deficit in T-maze spatial alternation, though both tasks emphasize memory for spatial location. Thus the explanation that delay-dependent effects observed in delayed alternation tasks following PL lesions occur because the correct response is based on spatial information is not persuasive. Discounting the spatial hypothesis is further supported by the observation that rats with PL lesion are not impaired in short-term memory of a spatial environment (Granon, Save, Buhot, \& Poucet, 1996; Poucet, 1989), spatial discrimination (Ragozzino, Detrick, \& Kesner, 1999), or place navigation (de Bruin, Sánchez-Santed, Heinsbroek, Donker, \& Postmen, 1994; Delatour \& Gisquet-Verrier, 2000; Granon \& Poucet, 1995).

Since the procedure used by Ragozzino et al. (1998) is basically a go/no-go task, it is also interesting to compare this task with traditional go/no-go tasks ( Delatour \& Gisquet-Verrier, 1996; Johnston et al., 1974; Winocur, 1991). In both types of tasks, rats have to select whether to run slow or fast depending on a previous response. Nevertheless, an additional ingredient of Ragozzino et al.'s (1998) task is that response selection is based on the spatial location of the target arm. Since the radial maze is considerably more complex than the simple runway used in the go/no-go task, it is possible that the overall pattern of a strong and delay-independent deficit observed by Ragozzino et al. results from an interaction between the complexity of the task (borne out by the number of alternative response choices) and the working memory requirement. In other words, the fact that working memory impairments following PL lesions can be observed even at short delays in the more complex situation suggests that this area might be particularly involved when the task requires both working memory and effortful processing.

The latter hypothesis is strongly supported by the results that rats with prelimbic lesion had serious and long-lasting difficulties in solving a spatial matching-to-sample task while they had at most a transitory impairment in a spatial non-matching-to-sample task (Granon et al., 1994). Although both tasks require the same amount of information to process, they differ by the type of response. Specifically, the matching-to-sample rule counteracts the rat's natural tendency to alternate, while the non-matching- 
to-sample rule reinforces this tendency. It is likely that the greater impairments observed in the former task result from the interaction between the effortful nature of the rule and the working memory requirement.

\section{The Radial Arm Maze Test}

If, as suggested by the previously reviewed evidence, the PL is involved in temporarily maintaining information in working memory when the task is made more difficult (e.g., when selection of the correct response must operate on a greater number of alternatives), then prelimbic lesions should result in large deficits in the radial arm maze task. As a matter of fact, in this task, rats have to find pieces of food located either at the end of each of the eight arms of the maze or in only four of them without visiting each arm more than once. Because the radial arm maze task involves both working memory and complex spatial response selection mechanisms, one expects that PL lesions will lead to profound deficits in the radial maze task. While some authors have found a clear performance deficit (Fritts et al., 1998; Seamans et al., 1995), others have found no impairment (Delatour \& GisquetVerrier, 1996; Joel et al., 1997). When an impairment is found, it is mainly due to a difficulty during the acquisition phase of the task and does not last more than 8 days (Fritts et al., 1998). Similarly, inserting a delay between the fourth and fifth choices of the animal results initially in a working memory impairment, which quickly recovers (Delatour \& Gisquet-Verrier, 1996). While some of these inconsistencies can be explained by subtle differences in lesion extent and procedural details, it remains that the observation of almost unaltered performance in the radial arm maze task poses a serious problem to the idea that the prelimbic function is primarily concerned with working memory.

However, the radial maze task has some peculiarities that might explain why prelimbic damage often does not produce a gross deficit (either delay dependent or delay independent) relative to spatial alternation tasks. One possibility might be that solving the radial arm maze task relies on the natural dynamic of the rat exploratory and foraging activity, since the rat solves the task by exploring the maze freely in one single trial. Even though the radial arm maze task certainly involves working memory processes, the use of lower cognitive processes not dependent on prefrontal activity may allow animals to achieve the task. This hypothesis receives some support from a recent unit recording study of the PL in rats subjected to the radial maze task. This study showed that very few neurons exhibited continuous activity during the delay between the first and subsequent visits of the arms (Jung, Qin, McNaughton, \& Barnes, 1998). Rather, the firing of prelimbic neurons correlated with specific behaviors or subcomponents of the task (approaching or leaving the goal, going to the center). This result represents a major difference with primate studies and does not support the view that, in this particular task, prefrontal neurons maintain "on-line" information (see Fuster, 1997, for review).
The different pieces of evidence reviewed so far allow us to propose the following general conclusions. First, the PL seems to have an intimate relationship with working memory. However, this relationship is more complex than a general involvement in all types of working memory tasks. Second, increasing the working memory delay does not consistently produce greater impairments in rats with prelimbic damage. Rather, the impairment will be enhanced if response selection is made more complex, for example, if it involves more alternative choices. One exception to this rule is the mostly unaltered performance of damaged rats in the radial arm maze task. However, our third conclusion might provide an explanation for this inconsistent result. In general, it is observed that the prelimbic damage-induced impairment is significantly increased when the task requires shifting from one strategy to another, whether the initial strategy has been learned (Granon \& Poucet, 1995; Ragozzino, Detrick, \& Kesner, 1999; Ragozzino, Wilcox, et al., 1999) or is spontaneously used by the animal (Granon et al., 1994). With regard to this point, solving the radial arm maze task might rely on unlearned, spontaneous strategies to a greater extent than other memory tasks (such as delayed alternation), thus making it less sensitive to prefrontal damage.

In short, the prefrontal deficit induced by prelimbic lesions does not appear as a "pure" working memory deficit. Rather, it results from a combination of factors relating to the overall difficulty of the memory task.

\section{PL AND ATTENTION}

As reviewed above, the rat PL seems to be involved in difficult working memory tasks that, presumably, require increased attentional capacities. While there are many human studies of the prefrontal cortex involvement in attention (e.g., Paus et al., 1997), comparable experiments in the rat have seldom been conducted. In addition, those studies have generally considered the MPFC as a whole. For example, selective neurochemical manipulations suggest some involvement of the mPFC in attentional mechanisms (Robbins et al., 1998). In addition, lesion studies suggest a role for the mPFC in divided attention. When the task requires allocation of resources to concurrent simultaneous information (Olton, Wenk, Church, \& Meck, 1988), rats with mPFC lesion cannot pay attention to several pieces of information at the same time in an operant task. Notably, such results might also reflect an impairment in selecting the appropriate response in relation to the context.

Nevertheless, a few studies have been aimed specifically at probing the attentional involvement of the PL. They have generally suggested a selective function in visual attention, for example when the animal must prepare itself to gather visual information that can occur at several locations (Muir, Everitt, \& Robbins, 1996). The task required the rat to respond to a brief stimulus (a light) that could randomly occur in one of the five possible po- 
sitions. Prelimbic lesions produce a strong deficit in this task. Interestingly, a comparable study in which rats with PL damage were tested with only two possible positions for the occurrence of the stimulus failed to report any significant impairment (Granon, Hardouin, Courtière, \& Poucet, 1998). This suggests that the task difficulty due to increased number of stimulus positions is an important factor for producing a prelimbic attentional deficit. Similarly, one might imagine that increasing the task difficulty by dissociating spatial positions for the stimulus and the response might also result in greater impairments. Lastly, another possibility might be to require the rat to constantly focus on a relevant event that can occur at any time. For example, in the second experiment of Granon et al.'s (1998) study, prelimbic damage was shown to produce long-lasting deficits when the rat was required to sustain its attention in order to detect a subtle change in light intensity. Since there was no explicit preparatory cue before the occurrence of such a change, the rat had to consistently focus on the light intensity. Since prelimbic lesions affect such detection, the PL is supposed to have a supervisory role in maintaining attention to relevant information (Bussey et al., 1997). Remarkably, rats with prelimbic lesions are not impaired in detecting spatial changes in their environment even when such changes are made less evident by increasing the complexity of the explored environment, a situation that presumably requires intact attentional capabilities (Granon et al., 1996). This result once again stresses the fact that the PL seems to be important for the learning of contingency rules that are not in the animal's natural behavioral repertoire or that counteract previously learned strategies, but much less so when behavior relies on spontaneous tendencies.

Interestingly, the above interpretation could provide an alternative explanation for many discrepancies in the literature. For example, while inactivations of the PL do not alter visual discrimination, response learning, or spatial learning (Joel, Weiner, \& Feldon, 1997; Seamans et al., 1995), they produce a strong impairment when the initially correct strategy has to be switched (Ragozzino, Detrick, \& Kesner, 1999; Ragozzino, Wilcox, et al., 1999). As an illustration, rats trained to use a spatial rule to get food face serious difficulties when they later have to use a response rule; this is also true for rats switched from the initial use of a response rule to a spatial rule (see also Dias, Robbins, \& Roberts, 1996, and Milner, 1963, for convergent results in monkeys and humans). It is reasonable to think that switching rules is a difficult task that requires effortful processing and attentional capacities. The aforementioned deficits can therefore be expected if an important function of the PL is to maintain attention to relevant information, especially when previously learned rules have to be ignored (Bussey et al., 1997; Granon et al., 1994; Li \& Shao, 1998; Ragozzino, Wilcox, et al., 1999). A similar reasoning could be used to explain why prelimbic lesions after training demonstrate much less dramatic working memory deficits than similar lesions made be- fore exposure to the learning situation (Granon et al., 1994).

\section{CONCLUSION}

This brief review suggests that, as in higher species, the PL of the rat is involved in an important manner in a variety of cognitive processes including attentional functions, working memory processes, and response-selection mechanisms. Within the prefrontal cortex, the prelimbic area seems to occupy a central position relative to other prefrontal subregions, but its exact function is still poorly understood. For example, while the PL is involved in a variety of working memory tasks, its role cannot be understood just in terms of its involvement in working memory. Specifically, results are inconsistent with the hypothesis that the PL is a simple temporary memory store since rats with prelimbic damage can be only marginally impaired in some working memory tasks and often are not sensitive to delay effects. In a similar vein, the PL memory function cannot be described by assuming that it is specific to a particular type of information. For example, spatial as well as nonspatial working memory can be seen to be impaired following prelimbic damage. Lastly, the involvement of the PL in attentional processes also is unclear. Rats with prelimbic damage can be unambiguously impaired in some attentional tasks, though quite unaffected in other tasks when subtle differences are introduced in the procedure.

Overall, the observation of a working memory impairment induced by damage to the PL appears to depend on the conjunction of several factors, which suggests that the PL function is complex and involves several mechanisms. Specifically, the PL is essential when the task demands are increased so that information processing is made more difficult, but much less so in tasks involving more automatic processing. This is true when increased difficulty relates to the perceptual aspects of the task, for example when detection of the relevant stimulus is made difficult either because of the possible number of positions (Granon et al., 1998; Muir et al., 1996) or because the task requires the rat to allocate continuous processing resources for stimulus detection (i.e., sustained attention, Granon et al., 1998). This is also true when the difficulty of the task is linked to its motor aspects. For instance, greater impairments are observed when response selection requires higher control. This occurs when the learning rule is not spontaneously used by the animal (e.g., Granon et al., 1994) or when it has been changed (e.g., Raggozino, Wilcox, et al., 1999).

Thus, although working memory deficits produced by prelimbic damage do result from poor temporarily organized memory (cf. Olton et al., 1988), they are associated with an additional impairment in effortful processing related to response selection. The prefrontal cortex is recruited when the task demands in cognitive processing are increased. This point merely suggests that the frontal cortical working memory system does not function sep- 
arately from other processing systems, such as the attentional processes required for acquiring complex associations. In both humans and rats, the prefrontal cortex appears to serve a special function that is most evident when the subject must adapt quickly to changing conditions in complex situations. Also, in both humans and rats, prefrontal damage results in impaired temporal structuring of information and mediation of prospective codesthat is, in the use of past experiences to set up expectancies and anticipations (Kesner, 1989; Kesner \& Holbrook, 1987; Petrides \& Milner, 1982). This in turn results in a reduced ability to plan complex sequences of behavior as a result of a failure to bridge temporally specific actions (Fuster, 1997).

While anatomical evidence for a homology between the rat prelimbic area and the primate DLPFC is far from convincing, functional similarities between these two cortical areas do exist. In both species, the prefrontal cortex seems to share some common function in those aspects of cognitive processing that, in humans, are usually referred to as executive functions. Within the rat prefrontal cortex, the prelimbic area appears to play a central role in such processes. This is not to say, however, that other prefrontal areas are not involved in such functions. Presumably, the prelimbic area is part of a larger network involving other prefrontal regions, such as the cingulate cortex, as well as subcortical structures (e.g., Floresco, Seamans, \& Phillips, 1997) whose functional specificity has yet to be elucidated. To conclude, the prefrontal cortex seems to be involved in similar processes in both primates and rats. Current evidence additionally suggests that the PL in the rat and the DLPFC in primates have a central role in these processes. We therefore believe that undermining the functions of the prefrontal cortex in the rat, and of the prelimbic area in particular, will greatly help understanding the prefrontal function in humans. Such an approach will be most fruitful if current methods used in cognitive neuropsychology and comparative psychology can be combined.

\section{REFERENCES}

Brito, G. N. O., \& BRITo, L. S. O. (1990). Septohippocampal system and the prelimbic sector of frontal cortex: A neuropsychological battery analysis in the rat. Behavioural Brain Research, 36, 127-146.

Brito, G. N. O., Thomas, G. J., \& Davis, B. J. (1982). Prelimbic cortex, mediodorsal thalamus, septum and delayed alternation in rats. Experimental Brain Research, 36, 127-146.

de Brabander, J. M., de Bruin, J. P. C., \& Van Eden, C. G. (1991). Comparison of the effects of neonatal and adult medial prefrontal cortex lesions on food hoarding and spatial delayed alternation. $B e$ havioural Brain Research, 42, 67-75.

de Bruin, J. P. C., Sánchez-Santed, F., Heinsbroek, R. P. W., Donker, A., \& Postmen, P. (1994). A behavioural analysis of rats with damage to the medial prefrontal cortex using Morris watermaze: Evidence for behavioural flexibility, but not for impaired spatial navigation. Brain Research, 652, 323-333.

Bussey, T. J., Muir, J. L., EveritT, B. J., \& Robbins, T. W. (1997). Triple dissociation of anterior cingulate, posterior cingulate and medial frontal cortices on visual discrimination tasks using a touchscreen testing procedure for the rat. Behavioral Neuroscience, 111, 920-936.
Condé, F., Audinat, E., Maire-Lepoivre, E., \& Crépel, F. (1990) Afferent connections of the medial frontal cortex of the rat: A study using retrograde transport of fluorescent dyes: I. Thalamic afferents. Brain Research Bulletin, 24, 341-354.

Condé, F., Maire-Lepoivre, E., Audinat, E., \& Crépel, F. (1995) Afferent connections of the medial prefrontal cortex of the rat: II. Cortical and sub-cortical afferents. Journal of Comparative Neurology, 352, 567-593.

Corbetta, M., Miezin, F. M., Dobmeyer, S., Shulman, G. L., \& PeTERSEN, S. E. (1991). Selective and divided attention during visual discriminations of shape, color, and speed: Functional anatomy by positron emission tomography. Journal of Neuroscience, 11, 23832402.

Delatour, B., \& Gisquet-Verrier, P. (1996). Prelimbic cortex specific lesions disrupt delayed-variable response tasks in the rat. $\mathrm{Be}$ havioral Neuroscience, 110, 1282-1298.

Delatour, B., \& Gisquet-Verrier, P. (1999). Lesions of the prelimbic and infralimbic cortices in rats do not disrupt response selection but induce delay-dependent deficits: Evidence for a role in working memory? Behavioral Neuroscience, 113, 941-955.

Delatour, B., \& Gisquet-Verrier, P. (2000). Functional role of the prelimbic-infralimbic cortices in spatial memory: Evidence for its involvement in attention and behavioural flexibility. Behavioural Brain Research, 109,113-128.

D'Esposito, M., Aguirre, G. K., Zarahn, E., Ballard, D., Shin, R. K., \& LEASE, J. (1998). Functional MRI studies of spatial and nonspatial working memory. Cognitive Brain Research, 7, 1-13.

Dias, R., Robrins, T. W., \& RoBerTs, A. C. (1996). Dissociation in prefrontal cortex of affective and attentional shift. Nature, 380, 69-72.

DuNNETT, S. B. (1990). Role of the prefrontal cortex and striatal output systems in short-term memory deficits associated with ageing, basal forebrain lesions, and cholinergic-rich grafts. Canadian Journal of Psychology, 44, 210-232.

Floresco, S. B., Seamans, J. K., \& Phillips, A. G. (1997). Selective roles for hippocampal, prefrontal cortical, and ventral striatal circuits in radial-arm maze tasks with or without a delay. Journal of Neuroscience, 17, 1880-1890.

Fritts, M. E., Asbury, E. T., Horton, J. E., \& IsaAC, W. L. (1998). Medial prefrontal lesion deficits involving or sparing the prelimbic area in the rat. Physiology \& Behavior, 64, 373-380.

Fuster, J. M. (1997). The prefrontal cortex: Anatomy, physiology, and neuropsychology of the frontal lobe. New York: Raven.

Goldman-Rakic, P. S. (1987). Circuitry of primate prefrontal cortex and regulation of behavior by representational memory. In F. Plum \& V. Mountcastle (Eds.), Handbook of physiology: Sec. I. Nervous system: Vol 5. Higher functions of the brain (pp. 373-417). Bethesda, MD: American Physiological Society.

Goldman-RAKIC, P. S. (1990). Cellular and circuit basis of working memory in prefrontal cortex of nonhuman primates. Progress in Brain Research, 85, 325-336.

Grafman, J. (1994). Alternative frameworks for the conceptualization of prefrontal lobe functions. In F. Boller \& J. Grafman (Eds.), Handbook of neuropsychology (Vol. 9, pp. 187-201). Amsterdam: Elsevier.

Granon, S., Hardouin, J., Courtière, A., \& Poucet, B. (1998). Evidence for the involvement of the rat prefrontal cortex in sustained attention. Quarterly, Journal of Experimental Psychology, 51B, 219 233.

Granon, S., \& Poucet, B. (1995). Medial prefrontal lesion in the rat and spatial navigation. Behavioral Neuroscience, 109, 474-484.

Granon, S., Save, E., Buhot, M.-C., \& Poucet, B. (1996). Effortful information processing in a spontaneous spatial situation by rats with medial prefrontal lesions. Behavioural Brain Research, 78, 147-154.

Granon, S., Vidal, C., Thinus-Blanc, C., Changeux, J.-P., \& PouCET, B. (1994). Working memory, response selection, and effortful processing in rats with medial prefrontal lesions. Behavioral Neuroscience, 108, 883-891.

Groenewegen, H. J., Berendse, H. W., Wolters, J. G., \& Lohman, A. H. M. (1990). The anatomical relationship of the prefrontal cortex with the striatopallidal system, the thalamus and the amygdala: Evidence for a parallel organization. Progress in Brain Research, 85, 95-118. 
JAY, T. M., GloWINSKI, J., \& ThIERRY, A. M. (1989). Selectivity of the hippocampal projection to the prelimbic area of the prefrontal cortex in the rat. Brain Research, 505, 337-340.

Joel, D., Tarrasch, R., Feldon, J., \& Weiner, I. (1997). Effects of electrolytic lesions of the medial prefrontal cortex or its subfields on 4-arm baited, 8-arm radial maze, two-way active avoidance and conditioned fear tasks in the rat. Brain Research, 765, 37-50.

JoEL, D., Weiner, I., \& Feldon, J. (1997). Electrolytic lesions of the medial prefrontal cortex in rats disrupt performance on an analog of the Wisconsin Card Sorting Test, but do not disrupt latent inhibition: Implications for animal models of schizophrenia. Behavioural Brain Research, 85, 187-201.

Johnston, V. S., HART, M., \& Howell, W. (1974). The nature of the medial wall deficit in the rat. Neuropsycholologia, 12, 497-503.

Jung, M. W., Qin, Y., McNaughton, B. L., \& Barnes, C. A. (1998). Firing characteristics of deep layer neurons in prefrontal cortex in rats performing spatial working memory tasks. Cerebral Cortex, 8, 437-450.

KESNER, R. P. (1989). Retrospective and prospective coding of information: Role of the medial prefrontal cortex. Experimental Brain Research, 74, 163-167.

KESNER, R. P., \& HolbrooK, T. (1987). Dissociation of item and order spatial memory in rats following medial prefrontal cortex lesions. Neuropsychologia, 25, 653-664.

KoLB, B. (1984). Functions of the prefrontal cortex of the rat: A comparative review. Brain Research Review, 8, 65-98.

KoLB, B. (1990). Prefrontal cortex. In B. Kolb \& R. C. Tees (Eds.), The cerebral cortex of the rat (pp. 437-458). Cambridge, MA: MIT Press.

Kolb, B., Buhrmann, K., McDonald, R., \& Sutherland, R. J. (1994). Dissociation of the medial prefrontal, posterior parietal, and posterior temporal cortex for spatial navigation and recognition memory in the rat. Cerebral Cortex, 6, 664-680.

Krettek, J. E., \& Price, J. L. (1977). The cortical projections of the mediodorsal nucleus and adjacent thalamic nuclei in the rat. Journal of Comparative Neurology, 171, 157-192.

LARSEN, J. K., \& DivaC, I. (1978). Selective ablations within the prefrontal cortex of the rat and performance of delayed alternation. Physiological Psychology, 6, 15-17.

LASHLEY, K. S. (1929). Brain mechanisms and intelligence. Chicago: University of Chicago Press.

Li, L., \& ShaO, J. (1998). Restricted lesions to ventral prefrontal subareas block reversal learning but not visual discrimination learning in rats. Physiology \& Behavior, 65, 371-379.

Miller, E. K. (1999). The prefrontal cortex: Complex neural properties for complex behavior. Neuron, 22, 15-17.

MiLner, B. (1963). Effects of different brain lesions on card sorting, the role of the frontal lobes. Archives of Neurology, 9, 90-100.

MILNER, B. (1971). Interhemispheric differences in the localization of psychological processes in man. British Medical Bulletin, 27, 272276.

Moscovitch, M., \& Winocur, G. (1995). Frontal lobes, memory and aging. In J. Grafman, K. J. Holyoak, \& F. Boller (Eds.), Structure and functions of the human prefrontal cortex (Annals of New York Academy of Sciences, Vol. 769, pp. 119-150). New York: New York Academy of Sciences.

Muir, J. L., Everitt, B. J., \& Robbins, T. W. (1996). The cerebral cortex of the rat and visual attentional function: Dissociable effects of mediofrontal, cingulate, anterior dorsolateral and parietal cortex lesions on a five-choice serial reaction time task. Cerebral Cortex, 6 , 470-481.

Ojemann, J. G., Buckner, R. L., Corbetta, M., \& Raichle, M. E. (1997). Imaging studies of memory and attention. Functional Imaging, 8, 307-319.

Olton, D. S., Wenk, G. L., Church, R. M., \& Meck, W. H. (1988). Attention and the frontal cortex as examined by simultaneous temporal processing. Neuropsychologia, 26, 307-318.

Owen, A. M., Herrod, N. J., Menon, D. K., Clark, J. C., Downey, S. P., Carpenter, A., Minhas, P. S., Turkheimer, F. E., Williams, E. J., Robbins, T. W., Sahakian, B. J., Petrides, M., \& Pickard, J. D. (1999). Redefining the functional organization of working memory processes within human lateral prefrontal cortex. European Journal of Neuroscience, 11, 567-574.

Paus, T., Zatorre, R. J., Hofle, N., Caramanos, Z., Gotman, J., Petrides, M., \& Evans, A. C. (1997). Time-related changes in neural systems underlying attention and arousal during the performance of an auditory vigilance task. Journal of Cognitive Neuroscience, 9, 392-408.

Petrides, M., \& Milner B. (1982). Deficits on subject-ordered tasks after frontal- and temporal-lobe lesions in man. Neuropsychologia, 20, 249-262.

Posner, M. I., \& Dehaene, S. (1994). Attentional networks. Trends in Neurosciences, 17, 75-79.

POUCET, B. (1989). Object exploration, habituation and response to a spatial change in rats following septal or medial frontal cortical damage. Behavioral Neuroscience, 103, 1009-1016.

PreUSS, T. (1995). Do rats have prefrontal cortex? The Rose-WoolseyAkert program reconsidered. Journal of Cognitive Neuroscience, 7 , $1-24$.

Pribram, K. H., Ahumada, A., Hartog, J., \& Ross, L. (1964). A progress report on the neurobiological processes disturbed by frontal lesions in primates. In J. M. Warren \& K. Akert (Eds.), The frontal granular cortex and behavior (pp. 28-55). New York: McGraw-Hill.

Ragozzino, M. E., Adams, S., \& Kesner, R. P. (1998). Differential involvement of the dorsal anterior cingulate and prelimbic-infralimbic areas of the rodent prefrontal cortex in spatial working memory. $B e$ havioral Neuroscience, 112, 293-303.

Ragozzino, M. E., Detrick, S., \& Kesner, R. P. (1999). Involvement of the prelimbic-infralimbic areas of the rodent prefrontal cortex in behavioral flexibility for place and response learning. Journal of Neuroscience, 19, 4585-4594.

Ragozzino, M. E., Wilcox, C., Raso, M., \& Kesner, R. P. (1999). Involvement of rodent prefrontal cortex subregions in strategy switching. Behavioral Neuroscience, 113, 32-41.

Rainer, G. AsaAd, W. F., \& Miller, E. K. (1998). Selective representation of relevant information by neurons in the primate prefrontal cortex. Nature, 393, 577-579.

RoBBins, T. W. (1997). Arousal systems and attentional processes. Biological Psychology, 45, 57-71.

Robbins, T. W., Granon, S., Muir, J. L., Durantou, F., Harrison, A. A., \& EvERITT, B. J. (1998). Neural systems underlying arousal and attention. In J. A. Harvey \& B. E. Kosofsky, Cocaine: Effects on the developing brain (Annals of the New York Academy of Sciences, Vol. 846, pp. 222-237). New York: New York Academy of Sciences.

SÁnchez-S anted, F., de Bruin, J. P. C., Heinsbroek, R. P. W., \& VERWER, R. W. H. (1997). Spatial delayed alternation of rats in a Tmaze: Effects of neurotoxic lesions of the medial prefrontal cortex and of T-maze rotations. Behavioural Brain Research, 84, 73-79.

Seamans, J. K., Floresco, S. B., \& Phillips, A. G. (1995). Functional differences between the prelimbic and anterior cingulate regions of the rat prefrontal cortex. Behavioral Neuroscience, 109, 1063-1073.

SesaCk, S. R., Deutsch, A. Y., Roth, R. H., \& Bunney, B. S. (1989). Topographical organization of the efferent projections of the medial prefrontal cortex in the rat: An anterograde study with Phaseolus vulgaris leucoagglutinin. Journal of Comparative Neurology, 290, 213 242.

STuss, D. T., \& BENSON, D. F. (1983). Frontal lobe lesions and behavior. In A. Kertesz (Ed.), Localization in neuropsychology (pp. 429. 454). New York: Academic Press.

Stuss, D. T., Shallice, T., Alexander, M. P., \& Picton, T. W. (1995). A multidisciplinary approach to anterior attentional functions. In J. Grafman, K. J. Holyoak, \& F. Boller (Eds.), Structure and functions of the human prefrontal cortex (Annals of the New York Academy of Science, Vol. 769, pp. 191-211). New York: New York Academy of Sciences.

Thomas, G. J., \& Briro, G. N. O. (1980). Recovery of delayed alternation in rats after lesions in medial frontal cortex and septum. Journal of Comparative \& Physiological Psychology, 94, 808-818.

Tzschenk, T. M., \& SChMiDT, W. J. (1998). Discrete quinolonic acid lesions of the rat prelimbic medial prefrontal cortex affect cocaineand MK-801-, but not morphine- and amphetamine-induced reward 
and psychomotor activation as measured with the place preference conditioning paradigm. Behavioural Brain Research, 97, 115-127.

Uylings, H. B. M., \& VAN EdEN, C. G. (1990). Qualitative and quantitative comparison of the prefrontal cortex in rats and in primates, including humans. Progress in Brain Research, 85, 31-62.

Van HaAren, F., de Bruin, J. P. C., Heinsbroek, R. P. W., \& Van de PoLL, N. E. (1985). Delayed spatial response alternation: Effects of delay-interval duration and lesions of the medial prefrontal cortex on response accuracy of male and female Wistar rats. Behavioural Brain Research, 18, 481-488.

WINOCUR, G. (1991). Functional dissociation of the hippocampus and prefrontal cortex in learning and memory. Psychobiology, 19, 11-20.

ZILLES, K. (1985). The cortex of the rat: A stereotaxic atlas. Berlin: Springer-Verlag.

\section{NOTE}

1. Another historical reason has to do with the widely accepted notion that the prefrontal cortex is intimately linked to intellectual faculties in hominids. Since the idea that rats might possess at least the precursors of such faculties has long been rejected, a comparitive approach of the neural systems involved in high-level processing was of course devoid of interest.

(Manuscript received October 14, 1999; revision accepted for publication February 23, 2000.) 